\title{
Calculation of total cross sections of multiple-sphere clusters
}

\author{
Daniel W. Mackowski \\ Department of Mechanical Engineering, Auburn University, Auburn, Alabama 36849
}

Received April 23, 1993; revised manuscript received June 23, 1994; accepted June 23, 1994

\begin{abstract}
A method for calculating the extinction, absorption, and scattering cross sections of clusters of neighboring spheres for both fixed and random orientations is developed. The analysis employs the superposition formulation for radiative interactions among spheres, in which the total field from the cluster is expressed as a superposition of vector spherical harmonic expansions about each of the spheres in the cluster. Through the use of addition theorems a matrix equation for the expansion coefficients is obtained. Further application of addition theorems on the inverse of the coefficient matrix is shown to yield analytical expressions for the orientation-averaged total cross sections of the sphere cluster. Calculations of the cross sections of pairs of spheres and fractal aggregates of several spheres are presented. It is found that a dipole representation of the field in each sphere does not adequately predict the absorption cross section of clusters of small-size-parameter spheres when the spheres are highly conducting. For this situation several multipole orders are required for an accurate calculation of the absorption cross section. In addition, the predicted absorption of sphere clusters can be significantly greater than that estimated from the sum of the isolated-sphere cross sections.
\end{abstract}

\section{INTRODUCTION}

Aerosol particles formed in combustion processes, such as carbonaceous soot, are frequently in the form of clusters of small, primary spheres. ${ }^{1}$ The radiative absorption and scattering properties of such particles are of key interest in the prediction of heat transfer rates from flames and the interpretation of laser diagnostic measurements. An accurate prediction of these properties, in turn, must account for the radiative interactions among the primary spheres in the cluster.

One approach to formulating the radiative behavior of a sphere cluster is to use the principle of superposition. Developed first by Brunning and $\mathrm{Lo}^{2}$ and later independently obtained by Borghese et al., ${ }^{3}$ this analysis constructs the scattered field from the cluster as a superposition of individual fields scattered from each of the spheres. The individual fields, in turn, are expressed in terms of expansions of vector spherical harmonics written about the origin of the sphere. By employing addition theorems, in which a harmonic about one origin can be expanded as harmonics about another origin, one obtains a system of equations for the scattered-field expansion coefficients for all the spheres in the cluster. Recent investigations of the radiative behavior of sphere clusters using the superposition formulation have been presented by Fuller and Kattawar, ${ }^{4-7}$ and recurrence relations for calculation of the addition coefficients have been developed by Mackowski. ${ }^{8}$

An alternative yet equivalent approach to the radiative analysis of sphere clusters begins with an integral formulation of Maxwell's equations. ${ }^{9}$ Jones, ${ }^{10}$ in a paper explicitly dealing with soot particles, used this formulation in conjunction with a Rayleigh-limit approximation of the field in each of the primary spheres to obtain a system of equations for the vector components of the electric field within each of the spheres in the cluster. Essentially, his approach is equivalent to a discrete-dipole model. ${ }^{11}$ Further application of the integral formulation to sphere clusters has been developed by Iskander et al. ${ }^{12}$ The reader is referred to a recent paper by $\mathrm{Ku}$ and Shim for a comparison of superposition and integral formulations as applied to soot particles. ${ }^{13}$

With regard to aerosols in general and soot aggregates in particular, the orientation-averaged absorption and scattering properties are ultimately desired. Exact analytical methods for calculating the cross sections of randomly oriented sphere clusters have been developed by Borghese et al. ${ }^{14}$ In the present paper an alternative formulation of the cross sections in both fixed and random orientations is developed. As we will see below, the methods offer some computational advantages in prediction of the cross sections.

\section{GENERAL FORMULATION}

The approach used here adopts the superposition solution. As I discussed in Section 1, the scattered electric field from the entire cluster is taken to be the superposition of scattered fields from each of the spheres in the cluster, i.e.,

$$
\mathbf{E}_{s}=\sum_{i}^{N_{s}} \mathbf{E}_{s}{ }^{i},
$$

where $N_{s}$ denotes the number of spheres in the cluster. The vector spherical harmonic expansion of each individual scattered field is written about the sphere origin as

$$
\begin{aligned}
\mathbf{E}_{s}{ }^{i}= & \sum_{n=1}^{\infty} \sum_{m=-n}^{n}\left[a_{m n}{ }^{i} \mathbf{N}_{m n}^{(3)}\left(k r^{i}, \theta^{i}, \phi^{i}\right)\right. \\
& \left.+b_{m n} \mathbf{M}_{m n}^{(3)}\left(k r^{i}, \theta^{i}, \phi^{i}\right)\right],
\end{aligned}
$$

where $k=2 \pi / \lambda$ is the wave number of the incident radiation having wavelength $\lambda, a_{m n}{ }^{i}$ and $b_{m n}{ }^{i}$ are expansion coefficients, and $\mathbf{N}_{m n}^{(3)}$ and $\mathbf{M}_{m n}^{(3)}$ are vector harmonics. ${ }^{15}$ 
The superscript (3) on the harmonics denotes that they are based on the spherical Hankel function $h_{n}(k r)$. The internal field in each sphere is represented by a similar expansion of the form

$$
\begin{aligned}
\mathbf{E}_{1}{ }^{i}= & \sum_{n=1}^{\infty} \sum_{m=-n}^{n}\left[d_{m n}{ }^{i} \mathbf{N}_{m n}^{(1)}\left(m^{i} k r^{i}, \theta^{i}, \phi^{i}\right)\right. \\
& \left.+c_{m n}{ }^{i} \mathbf{M}_{m n}^{(1)}\left(m^{i} k r^{i}, \theta^{i}, \phi^{i}\right)\right]
\end{aligned}
$$

where $m^{i}=n^{i}+i k^{i}$ denotes the complex refractive index of sphere $i$ and the superscript (1) denotes that the harmonics are now based on the spherical Bessel function $j_{n}(m k r)$.

Determination of the scattering coefficients $\left(a_{m n}{ }^{i}, b_{m n}{ }^{i}\right)$ for each sphere is the objective of the problem. We obtain equations for the coefficients by applying the boundary conditions for the electric and magnetic fields at the surface of each sphere in the cluster. The field arriving at each of the surfaces can be broken into two sources. The first consists of the external incident field that drives the scattering process in the first place. About a particular sphere $i$ the incident field can be expressed by the general expansion

$$
\begin{aligned}
\mathbf{E}_{0}{ }^{i}= & -\sum_{n=1}^{\infty} \sum_{m=-n}^{n}\left[p_{m n}{ }^{i} \mathbf{N}_{m n}^{(1)}\left(k r^{i}, \theta^{i}, \phi^{i}\right)\right. \\
& \left.+q_{m n}{ }^{i} \mathbf{M}_{m n}^{(1)}\left(k r^{i}, \theta^{i}, \phi^{i}\right)\right]
\end{aligned}
$$

where the coefficients $\left(p_{m n}{ }^{i}, q_{m n}{ }^{i}\right)$ are specified beforehand from the propagation direction and polarization of the incident field. The dependence of these coefficients on the incident direction and polarization will be discussed below.

The second source of incident radiation at the surface of sphere $i$ arises from radiation scattered from all other spheres in the cluster. To formulate the contribution of these sources into the boundary conditions, we must rewrite the expansions for the scattered fields in terms of vector harmonics about origin $i$. For the case $r^{i}<$ $R^{i j}$, where $R^{i j}$ denotes the distance from the centers of spheres $j$ and $i$, the addition theorem appears in the form

$$
\begin{aligned}
\mathbf{M}_{m n}^{(3)}\left(k r^{j}, \theta^{j}, \phi^{j}\right) & \\
= & \sum_{l=1}^{\infty} \sum_{k=-l}^{l}\left[A_{m n k l}^{(3)}\left(k R^{i j}, \Theta^{i j}, \Phi^{i j}\right) \mathbf{M}_{k l}^{(1)}\left(k r^{i}, \theta^{i}, \phi^{i}\right)\right. \\
& \left.+B_{m n k l}^{(3)}\left(k R^{i j}, \Theta^{i j}, \Phi^{i j}\right) \mathbf{N}_{k l}^{(1)}\left(k r^{i}, \theta^{i}, \phi^{i}\right)\right], \quad(5) \\
\mathbf{N}_{m n}^{(3)}\left(k r^{j}, \theta^{j}, \phi^{j}\right) & \\
= & \sum_{l=1}^{\infty} \sum_{k=-l}^{l}\left[A_{m n k l}^{(3)}\left(k R^{i j}, \Theta^{i j}, \Phi^{i j}\right) \mathbf{N}_{k l}^{(1)}\left(k r^{i}, \theta^{i}, \phi^{i}\right)\right. \\
& \left.+B_{m n k l}^{(3)}\left(k R^{i j}, \Theta^{i j}, \Phi^{i j}\right) \mathbf{M}_{k l}^{(1)}\left(k r^{i}, \theta^{i}, \phi^{i}\right)\right] . \quad(6)
\end{aligned}
$$

The addition coefficients $A$ and $B$ depend entirely on the distance and the direction of travel between $j$ and $i$, as contained in $k R^{i j}, \Theta^{i j}$, and $\Phi^{i j}$. The superscript (3) on the coefficients denotes that the coefficients are based on the spherical Hankel function. Were the situation that $r^{i}>R^{i j}$, the addition theorem would be given as

$$
\begin{aligned}
& \mathbf{M}_{m n}^{(3)}\left(k r^{j}, \theta^{j}, \phi^{j}\right) \\
&= \sum_{l=1}^{\infty} \sum_{k=-l}^{l}\left[A_{m n k l}^{(1)}\left(k R^{i j}, \Theta^{i j}, \Phi^{i j}\right) \mathbf{M}_{k l}^{(3)}\left(k r^{i}, \theta^{i}, \phi^{i}\right)\right. \\
&\left.\quad+B_{m n k l}^{(1)}\left(k R^{i j}, \Theta^{i j}, \Phi^{i j}\right) \mathbf{N}_{k l}^{(3)}\left(k r^{i}, \theta^{i}, \phi^{i}\right)\right], \quad(7) \\
& \mathbf{N}_{m n}^{(3)}\left(k r^{j}, \theta^{j}, \phi^{j}\right) \\
&= \sum_{l=1}^{\infty} \sum_{k=-l}^{l}\left[A_{m n k l}^{(1)}\left(k R^{i j}, \Theta^{i j}, \Phi^{i j}\right) \mathbf{N}_{k l}^{(3)}\left(k r^{i}, \theta^{i}, \phi^{i}\right)\right. \\
&\left.+B_{m n k l}^{(1)}\left(k R^{i j}, \Theta^{i j}, \Phi^{i j}\right) \mathbf{M}_{k l}^{(3)}\left(k r^{i}, \theta^{i}, \phi^{i}\right)\right], \quad(8)
\end{aligned}
$$

where the superscript (1) on the addition coefficients now indicates that the coefficients are based on the spherical Bessel function. Addition theorems for the harmonics $\mathbf{N}_{m n}^{(1)}$ and $\mathbf{M}_{m n}^{(1)}$ are given by the above two equations with $\mathbf{N}_{k l}^{(3)}$ and $\mathbf{M}_{k l}^{(3)}$ replaced by $\mathbf{N}_{k l}^{(1)}$ and $\mathbf{M}_{k l}^{(1)}$, respectively. In addition, the addition theorems for this case are uniformly convergent, regardless of the value of $R^{i j}$. Calculation of the addition coefficients with the use of recurrence relations is discussed in Appendix A.

By applying the field boundary conditions at each sphere and using the addition theorem given in Eqs. (5) and (6), one can obtain a linear relationship among the scattering coefficients of the spheres. After we truncate the scattered-field expansions for each sphere after a sufficient number of orders $n=N_{t}{ }^{i}$, the relationship can be expressed by the system of equations

$$
\begin{aligned}
a_{m n}{ }^{i}= & \bar{a}_{n}{ }^{i}\left\{p_{m n}{ }^{i}-\sum_{\substack{j=1 \\
j \neq i}}^{N_{s}} \sum_{l=1}^{N_{t}{ }^{j}} \sum_{k=-l}^{l}\left[A_{k l m n}^{(3)}\left(k R^{i j}, \Theta^{i j}, \Phi^{i j}\right) a_{k l}{ }^{j}\right.\right. \\
& \left.\left.+B_{k l m n}^{(3)}\left(k R^{i j}, \Theta^{i j}, \Phi^{i j}\right) b_{k l} j\right]\right\}, \\
b_{m n}{ }^{i}= & \bar{b}_{n}{ }^{i}\left\{q_{m n}{ }^{i}-\sum_{\substack{j=1 \\
j \neq i}}^{N_{s}} \sum_{l=1}^{N_{t}{ }^{j}} \sum_{k=-l}^{l}\left[A_{k l m n}^{(3)}\left(k R^{i j}, \Theta^{i j}, \Phi^{i j}\right) b_{k l}{ }^{j}\right.\right. \\
& \left.\left.+B_{k l m n}^{(3)}\left(k R^{i j}, \Theta^{i j}, \Phi^{i j}\right) a_{k l}^{j}\right]\right\} .
\end{aligned}
$$

Here $\bar{a}_{n}{ }^{i}$ and $\bar{b}_{n}{ }^{i}$ are the TM and TE Lorenz/Mie coefficients defined by ${ }^{15}$

$$
\begin{aligned}
& \bar{a}_{n}{ }^{i}=\frac{m^{i} \psi_{n}{ }^{\prime}\left(x^{i}\right) \psi_{n}\left(m^{i} x^{i}\right)-\psi_{n}\left(x^{i}\right) \psi_{n}{ }^{\prime}\left(m^{i} x^{i}\right)}{m^{i} \xi_{n}{ }^{\prime}\left(x^{i}\right) \psi_{n}\left(m^{i} x^{i}\right)-\xi_{n}\left(x^{i}\right) \psi_{n}{ }^{\prime}\left(m^{i} x^{i}\right)} \\
& \bar{b}_{n}{ }^{i}=\frac{\psi_{n}{ }^{\prime}(x) \psi_{n}\left(m^{i} x^{i}\right)-m^{i} \psi_{n}\left(x^{i}\right) \psi_{n}{ }^{\prime}\left(m^{i} x^{i}\right)}{\xi_{n}{ }^{\prime}\left(x^{i}\right) \psi_{n}\left(m^{i} x^{i}\right)-m^{i} \xi_{n}\left(x^{i}\right) \psi_{n}{ }^{\prime}\left(m^{i} x^{i}\right)},
\end{aligned}
$$

where $x^{i}=k a^{i}$ is the size parameter of sphere $i, \psi_{n}$ and $\xi_{n}$ are Ricatti-Bessel functions, and the prime denotes differentiation with respect to the argument.

In a compact form Eqs. (9) and (10) can be combined into

$$
\mathbf{a}_{m n p}{ }^{i}+\overline{\mathbf{a}}_{n p}{ }^{i} \sum_{\substack{j=1 \\ j \neq i}}^{N_{s}} \sum_{l=1}^{N_{t}{ }^{j}} \sum_{k=-l}^{l} \sum_{q=1}^{2} H_{m n p k l q}{ }^{i j} \mathbf{a}_{k l q}{ }^{j}=\overline{\mathbf{a}}_{n p}{ }^{i} \mathbf{p}_{m n p}{ }^{i} .
$$

The added subscripts $p$ and $q$ in Eq. (13) denote the mode (TM and TE, respectively) and take on the value 1 
or 2. Thus $\mathbf{a}_{m n 1}{ }^{i}=a_{m n}{ }^{i}$ and $\mathbf{a}_{m n 2}{ }^{i}=b_{m n}{ }^{i}$, and likewise for $\mathbf{p}_{m n p}{ }^{i}$ and $\overline{\mathbf{a}}_{n p}{ }^{i}$. The quantity $H$ represents the Hankel-function-based addition coefficients, with elements given by

$$
\begin{aligned}
& H_{m n 1 k l 1}{ }^{i j}=H_{m n 2 k l 2}{ }^{i j}=A_{k l m n}^{(3)}\left(k R^{i j}, \Theta^{i j}, \Phi^{i j}\right), \\
& H_{m n 1 k l 2}{ }^{i j}=H_{m n 2 k l 1}{ }^{i j}=B_{k l m n}^{(3)}\left(k R^{i j}, \Theta^{i j}, \Phi^{i j}\right) .
\end{aligned}
$$

The number of orders retained in the expansions, $N_{t}$, will generally depend on the size parameter of the spheres and their proximity to neighboring spheres. Determination of $N_{t}$ is discussed in Section 4 below. Also note that the total number of unknown scattering coefficients for each sphere (including order, degree, and mode), denoted $M^{i}$, will be given by

$$
M^{i}=2 N_{t}^{i}\left(N_{t}^{i}+2\right) .
$$

By formally inverting the system in Eq. (13) we can identify a transition matrix $T$ of the cluster of spheres such that

$$
\mathbf{a}_{m n p}{ }^{i}=\sum_{j=1}^{N_{s}} \sum_{l=1}^{N_{t}{ }^{j}} \sum_{k=-1}^{l} \sum_{q=1}^{2} T_{m n p k l q}{ }^{i j} \mathbf{p}_{k l q}{ }^{j} .
$$

As defined above, the $T$ matrix represents the individual scattered fields from each sphere in the cluster. This limits its usefulness in describing the scattered field from the cluster as a whole, and transformations will be described below that combine the superimposed fields from the cluster into a single field based on a single coordinate origin. Nevertheless, it will be advantageous to retain the sphere-centered formulation of the $T$ matrix in formulating the orientation-averaged cross sections of the cluster.

The representation of the scattered field from the cluster in a single expansion has been discussed by Borghese et $a l .{ }^{3}$ and Mackowski. ${ }^{8}$ The first step in the process is to identify an origin of the cluster. This could represent the average of the sphere positions but in general is arbitrary. The expansion coefficients for the incident field at the origin of sphere $i, \mathbf{p}^{i}$, can be obtained by a translation of the incident-field coefficients expanded about the cluster origin, $\mathbf{p}^{0}$, to origin $i$ through

$$
\mathbf{p}_{m n p}{ }^{i}=J_{m n p k l q}{ }^{i 0} \mathbf{p}_{k l q}{ }^{0},
$$

where the matrix $J$ is similar to $H$ given in Eqs. (14), except that the addition coefficients are now based on Bessel, rather than Hankel, functions. Above and in what follows, the summation over order, degree, and mode is implied for repeated subscripts. In a similar fashion the combined scattered field from all the spheres can be expressed as a single expansion written about the cluster origin by a translation of the fields from the spheres to the cluster origin. The expansion coefficients representing the total scattered field are thus given by

$$
\mathbf{a}_{m n p}{ }^{0}=\sum_{i=1}^{N_{s}} J_{m n p k l q}{ }^{0 i} \mathbf{a}_{k l q}{ }^{i} .
$$

Putting the preceding equations together, one obtains

$$
\begin{aligned}
\mathbf{a}_{m n p}{ }^{0} & =\sum_{i, j} J_{m n p m^{\prime} n^{\prime} p^{\prime}}{ }^{0 i} T_{m^{\prime} n^{\prime} p^{\prime} k^{\prime} l^{\prime} q^{\prime}}{ }^{i j} J_{k^{\prime} l^{\prime} q^{\prime} k l q}{ }^{j 0} \mathbf{p}_{k l q}{ }^{0} \\
& =T_{m n p k l q}{ }^{0} \mathbf{p}_{k l q}{ }^{0} .
\end{aligned}
$$

The cluster transition matrix $T^{0}$ treats the cluster of spheres as a single particle with a single origin; in this sense it is equivalent to the $T$ matrix obtained from integral/extended boundary condition method techniques. ${ }^{16,17}$ One might intuitively suspect that $T^{0}$ will typically be smaller in order than $T$ - since the fields from many origins have been boiled down to a single origin - but this is not generally the case. As noted by Borghese et al., ${ }^{14,18}$ the number of orders required for an accurate representation of both $\mathbf{p}^{0}$ and $\mathbf{a}^{0}$ increases with the distance from the original sphere origin to the cluster origin. With regard to Eqs. (17) and (18), this implies that the $J$ matrix is generally not square. For example, the number of columns in $J^{0 i}$ will be $M^{i}$ [where $M^{i}$ is defined in Eq. (15)], yet the number of rows will depend on the number of orders required for a reexpansion of the field from sphere $i$ about the cluster origin. Since translating the field expansion from its original origin to a second origin will generally increase the number of orders required for convergence of the expansion, the number of rows in $J^{0 i}$ will typically exceed the number of columns. Likewise, the number of columns in $J^{j 0}$ will exceed the number of rows. For clusters consisting of relatively few, widely spaced spheres the sphere-centered $T$ matrix would likely be smaller in order than the $T^{0}$ matrix. On the other hand, for a cluster of a large number of densely packed spheres the increase in order that is due to translation would be offset by the combination of the individual sphere expansions into a single expansion. In this case $T^{0}$ would be smaller in order than $T$.

\section{CROSS SECTIONS}

In situations in which the cross sections of the cluster for particular orientations are desired, it is advantageous to fix the positions of the spheres and rotate the direction and the polarization of the incident radiation to the desired configuration. In this way the $H$ matrix in Eq. (13) need only be calculated once for the given cluster geometry, and the properties can be obtained for multiple orientations by solution of Eq. (13) for various incident-field states. To specify the incident-field coefficients for an arbitrary direction and polarization, we take the plane incident wave to propagate in a direction $\mathbf{k}$ defined by a polar angle $\beta$ and an azimuthal angle $\alpha$ with respect to the cluster coordinates. The incident radiation is linearly polarized, with the electric vector at an angle $\gamma$ from the k- $z$ plane. Essentially, $\alpha, \beta$, and $\gamma$ define the Euler angles of rotation of the incident field from a $z$ propagating, $x$-polarized state. ${ }^{19}$ The incident-field coefficients at sphere $i$ can then be expanded by

$$
\begin{aligned}
\left(p_{m n}^{i}, q_{m n}{ }^{i}\right)= & \left(p_{m n}, q_{m n}\right) \exp i\left[Z^{i} \cos \beta+(\sin \beta)\right. \\
& \left.\times\left(X^{i} \cos \alpha+Y^{i} \sin \alpha\right)\right], \\
p_{m n}= & -i^{n+1} \frac{1}{E_{m n}}\left[\tau_{m n}(\beta) \cos \gamma-i \pi_{m n}(\beta) \sin \gamma\right] \\
& \times \exp (-i m \alpha), \\
q_{m n}= & -i^{n} \frac{1}{E_{m n}}\left[\tau_{m n}(\beta) \sin \gamma+i \pi_{m n}(\beta) \cos \gamma\right] \\
& \times \exp (-i m \alpha),
\end{aligned}
$$

where $X^{i}, Y^{i}$, and $Z^{i}$ denote the origin of $i$ with respect 
to the cluster origin,

$$
\begin{gathered}
E_{m n}=\frac{n(n+1)}{2 n+1} \frac{(n+m) !}{(n-m) !}, \\
\tau_{m n}(\beta)=\frac{\mathrm{d}}{\mathrm{d} \beta} P_{n}{ }^{m}(\cos \beta),
\end{gathered}
$$

In Eqs. (22), $P_{n}{ }^{m}$ is the associated Legendre function.

For the given state of the incident radiation the extinction cross section of sphere $i$ in the cluster is obtained from the optical theorem as ${ }^{8,15}$

$$
C_{\mathrm{ext}}^{i}=\frac{4 \pi}{k^{2}} \operatorname{Re}\left(E_{m n} \mathbf{P}_{m n p}^{i *} \mathbf{a}_{m n p}{ }^{i}\right)
$$

where the superscript $*$ denotes the conjugate. From conservation of energy, the total extinction cross section of the cluster is the sum of the individual sphere extinction cross sections for all spheres in the cluster, i.e.,

$$
C_{\mathrm{ext}}=\sum_{i=1}^{N_{s}} C_{\mathrm{ext}}{ }^{i}
$$

Alternatively, the extinction cross section of the cluster can be obtained from the single scattered-field expansion given in Eq. (18) by ${ }^{3}$

$$
C_{\text {ext }}=\frac{4 \pi}{k^{2}} \operatorname{Re}\left(E_{m n} \mathbf{P}_{m n p}{ }^{0 *} \mathbf{a}_{m n p}{ }^{0}\right) .
$$

By using Eq. (19) in Eq. (25), one obtains

$$
C_{\mathrm{ext}}=\frac{4 \pi}{k^{2}} \operatorname{Re}\left(E_{m n} \mathbf{p}_{m n p}{ }^{0 *} T_{m n p k l q}{ }^{0} \mathbf{p}_{k l q}{ }^{0}\right)
$$

The orientation-averaged extinction cross section of the cluster can now be obtained by an integration of Eq. (26) over all incident directions and polarizations. To do this, we employ the integral relationship

$$
\begin{aligned}
\frac{1}{8 \pi^{2}} \int_{0}^{2 \pi} \int_{0}^{\pi} \int_{0}^{2 \pi} \mathbf{p}_{m n p}{ }^{*} \mathbf{p}_{k l q} \mathrm{~d} \gamma & \cos \beta \mathrm{d} \beta \mathrm{d} \alpha \\
& =\frac{1}{2 E_{m n}} \delta_{k m} \delta_{l n} \delta_{p q}
\end{aligned}
$$

where $\delta$ is the Kroneker delta function. Consequently, the orientation-averaged extinction cross section is

$$
\begin{aligned}
\bar{C}_{\mathrm{ext}} & =\frac{2 \pi}{\hbar^{2}} \operatorname{Re} T_{m n p m n p}{ }^{0} \\
& =\frac{2 \pi}{\hbar^{2}} \operatorname{Re}\left(\operatorname{Tr} T^{0}\right) .
\end{aligned}
$$

The above result has been obtained by Borghese et al. ${ }^{14}$ (who used a somewhat different approach) for the specific case of sphere clusters as well as by Khlebtsov ${ }^{20}$ and Mishchenko ${ }^{21}$ for the case of general nonspherical particles described by a $T^{0}$ matrix.

Obviously, to exploit the result in Eq. (28) it is necessary to transform the sphere-centered $T$ matrix into the cluster-centered $T^{0}$ matrix by means of Eq. (19). As we mentioned in Section 2 above, this can significantly increase the order of the expansions when the spheres are separated by large relative distances. For these situations it would be advantageous to retain the spherecentered formulation of the analysis in computing the orientation-averaged cross sections. To do this, we first substitute Eq. (19) into Eq. (28), resulting in

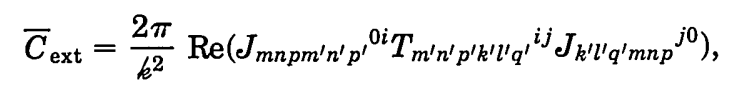

where the sum over the sphere indices $i$ and $j$ is implied. The above equation is reduced by the introduction of some properties of the $J$ translation matrix. First, the conjugate of a $J$ matrix is related by

$$
E_{m n} J_{m n p k l q}^{i j^{*}}=E_{k l} J_{k l q m n p}^{j i}
$$

Also, a translation from $i$ to $j^{\prime}$ and then from $j^{\prime}$ to $j$ is equivalent to a translation directly from $i$ to $j$. This leads to

$$
\sum_{n^{\prime}=1}^{\infty} \sum_{m^{\prime}=-n^{\prime}}^{n^{\prime}} \sum_{p^{\prime}=1}^{2} J_{m n p m^{\prime} n^{\prime} p^{\prime}} j^{\prime} J_{m^{\prime} n^{\prime} p^{\prime} k l q}^{j^{\prime} i}=J_{m n p k l q}{ }^{j i} .
$$

We have explicitly written the summations in Eq. (31) to emphasize that the identity is exact only in the limit $n^{\prime} \rightarrow \infty$. Comparing the above relation with Eq. (29), one sees that the summation over $m$ and $n$ can be analytically taken to infinity. This results in

$$
\bar{C}_{\mathrm{ext}}=\frac{2 \pi}{k^{2}} \operatorname{Re}\left(J_{k l q m n p}^{j i} T_{m n p k l q}^{i j}\right) .
$$

It is important to realize that the number of rows and columns in the matrix $J^{j i}$ will be $M^{j}$ and $M^{i}$. Thus the size of $J^{j i}$ is fixed by the number of orders retained in the expansions about spheres $i$ and $j$ and is not dependent on the distance between $i$ and $j$. Consequently, the above formulation for the orientation-averaged extinction cross section eliminates the problems associated with reexpanding the fields about a common origin and the ensuing increase in expansion order. By using the relationship for $J$ in Eq. (30) and noting that $J_{m n p k l q}{ }^{i i}=\delta_{k m} \delta_{l n} \delta_{p q}$, we obtain the following equivalent expression for $\bar{C}_{\text {ext }}$ :

$$
\begin{aligned}
\bar{C}_{\text {ext }}= & \frac{2 \pi}{k^{2}} \operatorname{Re}\left[T_{m n p m n p}{ }^{i i}+\left(J_{m n p k l q}^{i j} T_{k l q m n p}{ }^{j i}\right.\right. \\
& \left.\left.+\frac{E_{k l}}{E_{m n}} J_{k l q m n p}{ }^{i j *} T_{k l q m n p} i j\right)\right], \quad j>i .
\end{aligned}
$$

In Eq. (33) it is understood that $i$ and $j$ go from 1 to $N_{s}$ and $i+1$ to $N_{s}$, respectively. From a numerical point of view, Eq. (33) is better suited than Eq. (32) for calculating $\bar{C}_{\text {ext }}$, in that the matrices $J^{i j}$ need only be calculated for $j>i$.

The scattering cross section of the cluster for a particular incident field is given by

$$
C_{\text {sca }}=\frac{4 \pi}{\hbar^{2}} E_{m n} \mathbf{a}_{m n p}{ }^{0 *} \mathbf{a}_{m n p}{ }^{0} .
$$

The above formulation, while it is conceptually simple, is often not the best way to calculate the scattering cross section. As was the case in Eq. (25), it requires the transformation of the sphere-centered scattered fields into a single expansion. However, using the properties of the 
$J$ matrix developed above, one can formulate expressions for the scattering cross section, in both fixed and random orientations, that retain the sphere-centered expansions. By substituting Eq. (18) into Eq. (34) and using the relations given in Eqs. (30) and (31), one obtains

$$
C_{\text {sca }}=\frac{4 \pi}{k^{2}} \operatorname{Re}\left(E_{k l} \mathbf{a}_{k l q}{ }^{i *} J_{k j q m n p}^{i j} \mathbf{a}_{m n p}{ }^{j}\right) .
$$

Expanding further, we reduce Eq. (35) to

$$
\begin{aligned}
C_{\mathrm{sca}}= & \frac{4 \pi}{k^{2}} E_{m n}\left[\mathbf{a}_{m n p}{ }^{i *} \mathbf{a}_{m n p}{ }^{i}\right. \\
& \left.+2 \operatorname{Re}\left(\mathbf{a}_{m n p}{ }^{i *} J_{m n p k j q}^{i j} \mathbf{a}_{k l q}^{j}\right)\right], \quad j>i .
\end{aligned}
$$

As we can see, the above formulation involves only the individual sphere expansions. In addition, the two terms in Eq. (36) can be recognized as the independent (singlesphere) and dependent (multiple-sphere) contributions to total scattering from the cluster. A similar equation for the scattering cross section of the cluster has been obtained by Fuller.7

To obtain the random-orientation scattering cross section, we use Eqs. (16) and (17) in Eq. (35) to eliminate a. The incident field is then integrated over all incident directions through Eq. (27), and the $J$ properties in Eq. (31) are again put to use. This results in

$$
\begin{aligned}
\bar{C}_{\text {sca }}= & \frac{2 \pi}{k^{2}} \operatorname{Re}\left\{\frac { E _ { m n } } { E _ { k l } } \left[J_{m n p u v w} i i^{\prime} T_{u v w k l q}{ }^{i^{\prime} j}\right.\right. \\
& \left.\left.\times\left(T_{m n p u^{\prime} v^{\prime} w^{\prime}}^{i j^{\prime}} J_{u^{\prime} v^{\prime} w^{\prime} k l q}^{j^{\prime} j}\right)^{*}\right]\right\} .
\end{aligned}
$$

We can expand Eq. (37) in terms of the $T^{0}$ matrix to yield

$$
\bar{C}_{\mathrm{sca}}=\frac{2 \pi}{\hbar^{2}} \frac{E_{m n}}{E_{k l}}\left(T_{m n p k l q}{ }^{0} T_{m n p k l q}{ }^{0 *}\right),
$$

which again is in agreement with general relations for the $T^{0}$ matrix. ${ }^{20,21}$

Traditionally, one uses analytical methods to determine the extinction and scattering cross sections, and the absorption cross section $C_{\text {abs }}$ is obtained simply from their difference. With regard to the sphere clusters, it is shown below that it is actually advantageous to determine $C_{\text {abs }}$ directly-and to use it along with $C_{\text {ext }}$ to determine $C_{\text {sca. }}$.

For a particular orientation the absorption cross section for each sphere can be obtained from an integration of the normal component of the internal radiant intensity at the sphere surface over the area of the sphere by means of

$$
\begin{aligned}
C_{\mathrm{abs}}{ }^{i}= & \frac{a^{i 2}}{I_{0}} \int_{0}^{2 \pi} \int_{0}^{\pi} I_{1}\left(r=a^{i}\right) \sin \theta \mathrm{d} \theta \mathrm{d} \theta \phi \\
= & \frac{a^{i 2}}{2 I_{0}} \operatorname{Re}\left[\left.\int_{0}^{2 \pi} \int_{0}^{\pi}\left(E_{1 \theta} H_{1 \phi}{ }^{*}-E_{1 \phi} H_{1 \theta}{ }^{*}\right)\right|_{r=a^{i}}\right. \\
& \times \sin \theta \mathrm{d} \theta \mathrm{d} \phi]
\end{aligned}
$$

where $H$ denotes the magnetic field. By direct integration of the internal field expansions and a bit of algebraic manipulation the following expression is ultimately obtained $^{8}$ :

$$
C_{\mathrm{abs}}{ }^{i}=\frac{4 \pi}{h^{2}} \sum_{n=1}^{N_{t}{ }^{i}} \sum_{m=-n}^{n} E_{m n}\left(\bar{d}_{n}{ }^{i}\left|a_{m n}{ }^{i}\right|^{2}+\bar{c}_{n}{ }^{i}\left|b_{m n}{ }^{i}\right|^{2}\right)
$$

where the real-valued quantities $\bar{d}$ and $\bar{c}$ are given by

$$
\begin{aligned}
\bar{d}_{n}{ }^{i} & =\frac{\operatorname{Re}\left[i \psi_{n}{ }^{\prime}\left(m^{i} x^{i}\right) \psi_{n}{ }^{*}\left(m^{i} x^{i}\right) m^{i *}\right]}{\left|m^{i} \psi_{n}\left(m^{i} x^{i}\right) \psi_{n}{ }^{\prime}\left(x^{i}\right)-\psi_{n}\left(x^{i}\right) \psi_{n}{ }^{\prime}\left(m^{i} x^{i}\right)\right|^{2}}, \\
\bar{c}_{n}{ }^{i} & =\frac{\operatorname{Re}\left[i \psi_{n}{ }^{\prime}\left(m^{i} x^{i}\right) \psi_{n}{ }^{*}\left(m^{i} x^{i}\right) m^{i}\right]}{\left|\psi_{n}\left(m^{i} x^{i}\right) \psi_{n}{ }^{\prime}\left(x^{i}\right)-m^{i} \psi_{n}\left(x^{i}\right) \psi_{n}{ }^{\prime}\left(m^{i} x^{i}\right)\right|^{2}}
\end{aligned}
$$

The cluster absorption cross section is simply the sum of the individual sphere absorption cross sections, i.e.,

$$
C_{\mathrm{abs}}=\sum_{i=1}^{N_{s}} C_{\mathrm{abs}}{ }^{i}
$$

In the condensed form the absorption cross section of the cluster is

$$
C_{\mathrm{abs}}=\frac{4 \pi}{k^{2}} E_{m n} \overline{\mathbf{d}}_{n p}{ }^{i} \mathbf{a}_{m n p}{ }^{i} \mathbf{a}_{m n p}{ }^{i *},
$$

where $\overline{\mathbf{d}}_{n 1}=\bar{d}_{n}, \overline{\mathbf{d}}_{n 2}=\bar{c}_{n}$, and the sum over subscripts and superscripts is again implied. As one can see by a comparison of Eqs. (36) and (44), direct calculation of the absorption cross section can be performed with considerably less computational effort than direct calculation of the scattering cross section, in that the expression for the absorption cross section does not explicitly contain a dependent scattering contribution. As a result, it is much more efficient to calculate $C_{\text {ext }}$ and $C_{\text {abs }}$ directly and to determine $C_{\text {sca }}$ from their difference. Of course, if the spheres are nonabsorbing, Eq. (44) will be identically zero (since $\overline{\mathbf{d}}$ will be zero), for which $C_{\text {sca }}=C_{\text {ext }}$.

The orientation-averaged absorption cross section is obtained by application of the same approach used above with $\bar{C}_{\text {ext }}$ and $\bar{C}_{\text {sca. }}$. The result is

$$
\bar{C}_{\mathrm{abs}}=\frac{2 \pi}{\hbar^{2}} \overline{\mathbf{d}}_{n p}{ }^{i} \frac{E_{m n}}{E_{k^{\prime} l^{\prime}}} \operatorname{Re}\left(T_{m n p k l q}^{i j} J_{k l q k^{\prime} l^{\prime} q^{\prime}}{ }^{j j^{\prime}} T_{m n p k^{\prime} l^{\prime} q^{\prime}}{ }^{i j^{\prime} *}\right),
$$

which can be reduced to

$$
\begin{aligned}
& \bar{C}_{\mathrm{abs}}=\frac{2 \pi}{\iota^{2}} \overline{\mathbf{d}}_{n p}{ }^{i} \frac{E_{m n}}{E_{k l}}\left[T_{m n p k l q}^{i j} T_{m n p k l q}^{i j *}\right. \\
& +2 \operatorname{Re}\left(T_{m n p k^{\prime} l^{\prime} q^{\prime}}^{i j} J_{k^{\prime} l^{\prime} q^{\prime} k l q}^{j j^{\prime}} T_{\left.\left.m n p k l q^{i j^{\prime} *}\right)\right],}\right. \\
& j^{\prime}>j \text {. }
\end{aligned}
$$

Note that computation of $\bar{C}_{\text {abs }}$ by means of Eq. (46) involves one fewer matrix multiplication than calculation of $\bar{C}_{\text {sca }}$ from Eq. (37).

\section{RESULTS AND DISCUSSION}

To test the veracity of the formulation, we compared calculation results against previously published experimental measurements of Wang et al. ${ }^{22}$ The experimental system consisted of a pair of spheres, with incident radiation propagating along the axis connecting the two spheres. 


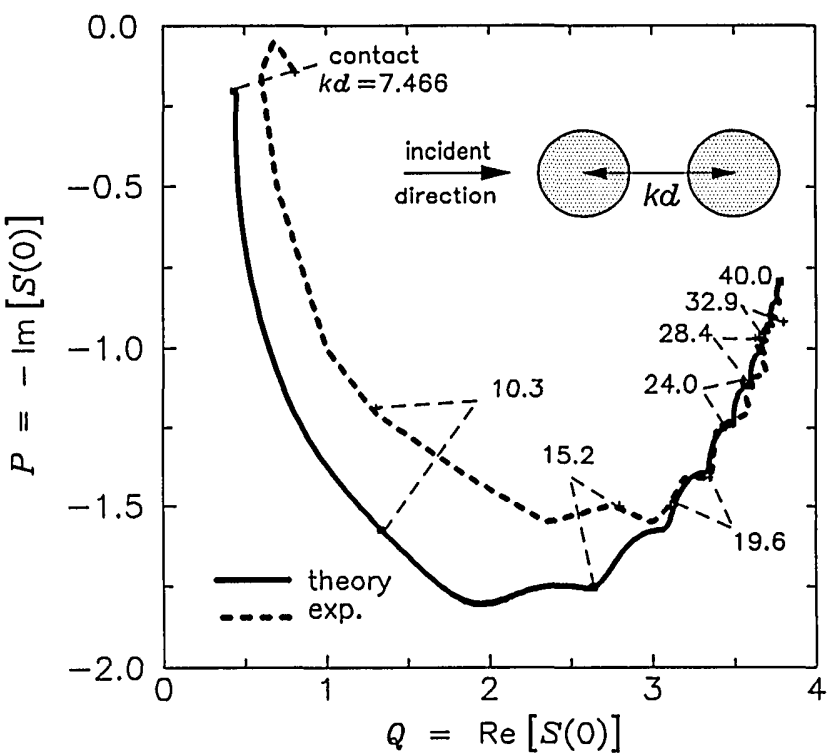

Fig. 1. Comparison of theoretical predictions of complex forward-scattering amplitude for a two-sphere cluster with the experimental measurements of Wang et al. ${ }^{22}$

Using a microwave approach, Wang et al. measured the magnitude and the phase of the forward-scattered radiation, from which the complex forward-scattering amplitude $S(0)$ could be inferred. We make a comparison with the present analysis by noting that, from Eq. (23), the dimensionless forward-scattering amplitude is given by

$$
S(0)=\frac{4 \pi}{k^{2} G} \sum_{i=1}^{N_{s}} E_{m n} \mathbf{p}_{m n p}{ }^{i *} \mathbf{a}_{m n p}{ }^{i}
$$

where $G$ is the sum of the geometrical cross sections of the two spheres. The calculations for the two-sphere system were carried out by solution of Eq. (13) for an incident field given by $\alpha=\beta=\gamma=0$. In general, the two-sphere system offers a considerable reduction in the order of the equations, provided that the spheres are situated so that they share a common $z$ axis. In this configuration the azimuthal modes are decoupled, and the equations can be solved sequentially for each azimuthal mode $m$. In addition, the case $\beta=0$ results in a further simplification of the problem, in that the incident-field expansion coefficients $\mathbf{p}_{m n p}$ will be zero except for $m= \pm 1$.

The comparison of experimental and theoretical results is given in Fig. 1, where the imaginary part of $S(0)$ is plotted versus the real part (which corresponds to the extinction efficiency $Q_{\text {ext }}$ ), with separation distance $k d$ as a parameter. Note that the theoretical results track fairly well the experimental results. A likely cause of the difference between experiment and theory is uncertainty in the sphere refractive index.

An identical comparison between the Wang et al. data and theory was made by Borghese et al., ${ }^{18}$ and the theoretical results presented here appear to agree exactly with the previous calculations. However, the approach of Borghese et al. was based on reexpansion of the scattered fields from the spheres about a common origin of the cluster, leading to Eq. (25). As those authors noted, this approach resulted in numerical problems as $k d$ increased because of the high number of orders required in the expansion. These difficulties prevented them from extending their calculations beyond a maximum value of $k d$. In contrast, the approach used in Eq. (23), in which the sphere-centered expansions are retained, results in no numerical problems for increasing $k d$. Indeed, as the spheres become more separated, the number of required scattering orders for each sphere actually decreases, which is due to the decreasing field coupling between the spheres.

The required number of orders in the expansions for the scattered fields is an issue that had not been addressed in detail in previous theoretical investigations of neighboring-sphere scattering. Borghese et al. ${ }^{14,18}$ noted that, in reexpansion of the fields about a common origin of the cluster, the number of orders required for convergence was proportional to the size parameter based on the maximum dimension of the cluster. In the present paper, in which the sphere-centered expansions are retained, one expects the number of orders for each sphere to be proportional to the size parameter of the sphere. Indeed, results show that, for most cases, the criterion developed by Wiscombe ${ }^{23}$ and modified by Bohren and Huffman ${ }^{15}$ will provide a good, conservative estimate of the required number of orders. This criterion is given as ${ }^{15}$

$$
N_{t}^{i} \approx x^{i}+4\left(x^{i}\right)^{1 / 3}+2 .
$$

Surprisingly enough, the results indicate that the one situation in which the above criterion fails is the combination of touching spheres having size parameters significantly less than unity and relatively large real and imaginary parts of the refractive index. Results of calculations that illustrate this behavior are shown in Table 1, where the extinction and absorption cross sections of clusters of two identical touching spheres, normalized by that predicted from Lorenz/Mie theory for isolated spheres, are given for various sphere size parameters and refractive indices and three states of the incident radiation. The case $\beta=\gamma=0^{\circ}$ has the incident radiation parallel to the pair axis, $\beta=90^{\circ}$ and $\gamma=0^{\circ}$ has the radiation perpendicular to the axis and polarized parallel to the axis, and $\beta=90^{\circ}$ and $\gamma=90^{\circ}$ is the same as for the previous case, except that the polarization is perpendicular to the axis. The last column in the table lists the number of expansion orders that we required to achieve cross sections that were within $0.5 \%$, on a relative basis of the converged $\left(N_{t} \rightarrow \infty\right)$ result. For the first refractive index $(m=1.6+0.6 i)$ and small size parameters $(x=0.1)$ the results indicate that the spheres behave almost as dipoles. Indeed, calculations that retained only the first electric dipole term for these cases resulted in cross sections that were only a few percent smaller than the listed values, which include the first two electric and magnetic orders. As one would expect, the cross sections for the $\beta=90^{\circ}$ and $\gamma=0^{\circ}$ configuration are appreciably larger than those for two other cases when the size parameters are small. For this situation the pair, when it is aligned with the electric-field vector, is polarized to a greater extent than when it is aligned perpendicular to the field. The same behavior is predicted for prolate spheroids and infinite-length cylinders in the Rayleigh limit.

For spheres having a larger magnitude of $m^{2}$ the results show that we require a surprisingly large num- 
Table 1. Results for a Cluster of Two Identical Touching Spheres ${ }^{a}$

\begin{tabular}{|c|c|c|c|c|c|c|}
\hline$x$ & $m$ & $\beta$ & $\gamma$ & $C_{\text {ext }} / C_{\text {ext }, i}$ & $C_{\mathrm{abs}} / C_{\mathrm{abs}, i}$ & $N_{t}^{b}$ \\
\hline 0.10 & $1.6+0.6 i$ & 0 & 0 & 0.915 & 0.914 & 2 \\
\hline 0.10 & $1.6+0.6 i$ & 90 & 0 & 1.272 & 1.271 & 2 \\
\hline 0.10 & $1.6+0.6 i$ & 90 & 90 & 0.913 & 0.913 & 2 \\
\hline 1.00 & $1.6+0.6 i$ & 0 & 0 & 1.067 & 1.067 & 3 \\
\hline 1.00 & $1.6+0.6 i$ & 90 & 0 & 1.030 & 0.907 & 3 \\
\hline 1.00 & $1.6+0.6 i$ & 90 & 90 & 0.856 & 0.810 & 3 \\
\hline 10.00 & $1.6+0.6 i$ & 0 & 0 & 0.612 & 0.592 & 14 \\
\hline 10.00 & $1.6+0.6 i$ & 90 & 0 & 0.981 & 0.966 & 14 \\
\hline 10.00 & $1.6+0.6 i$ & 90 & 90 & 0.995 & 1.015 & 14 \\
\hline 0.10 & $2.0+1.0 i$ & 0 & 0 & 0.878 & 0.877 & 2 \\
\hline 0.10 & $2.0+1.0 i$ & 90 & 0 & 1.576 & 1.574 & 5 \\
\hline 0.10 & $2.0+1.0 i$ & 90 & 90 & 0.876 & 0.875 & 2 \\
\hline 1.00 & $2.0+1.0 i$ & 0 & 0 & 1.059 & 1.088 & 3 \\
\hline 1.00 & $2.0+1.0 i$ & 90 & 0 & 0.986 & 0.869 & 3 \\
\hline 1.00 & $2.0+1.0 i$ & 90 & 90 & 0.802 & 0.770 & 3 \\
\hline 10.00 & $2.0+1.0 i$ & 0 & 0 & 0.613 & 0.600 & 14 \\
\hline 10.00 & $2.0+1.0 i$ & 90 & 0 & 0.977 & 0.962 & 14 \\
\hline 10.00 & $2.0+1.0 i$ & 90 & 90 & 0.994 & 1.032 & 14 \\
\hline 0.10 & $3.0+2.0 i$ & 0 & 0 & 0.845 & 0.842 & 2 \\
\hline 0.10 & $3.0+2.0 i$ & 90 & 0 & 2.546 & 2.541 & 10 \\
\hline 0.10 & $3.0+2.0 i$ & 90 & 90 & 0.842 & 0.839 & 2 \\
\hline 1.00 & $3.0+2.0 i$ & 0 & 0 & 1.014 & 1.196 & 3 \\
\hline 1.00 & $3.0+2.0 i$ & 90 & 0 & 0.948 & 0.916 & 6 \\
\hline 1.00 & $3.0+2.0 i$ & 90 & 90 & 0.746 & 0.739 & 3 \\
\hline 10.00 & $3.0+2.0 i$ & 0 & 0 & 0.618 & 0.629 & 14 \\
\hline 10.00 & $3.0+2.0 i$ & 90 & 0 & 0.975 & 0.967 & 14 \\
\hline 10.00 & $3.0+2.0 i$ & 90 & 90 & 0.991 & 1.055 & 14 \\
\hline
\end{tabular}

${ }^{a}$ Cross sections are divided by the result obtained for isolated Lorenz/Mie spheres.

${ }^{b}$ Number of orders required for a less than $0.5 \%$ relative residual error in series expansions.

ber of expansion orders to resolve the extinction and absorption cross sections of small-size-parameter spheres. For example, a pair of $x=0.1$ and $m=2+1 i$ spheres aligned parallel to the incident electric vector requires 5 orders, and for $m=3+2 i$ the number goes up to 11 . Note that $N_{t}$ is actually larger for $x=0.1$ than it is for $x=1$. One can explain this behavior, which is somewhat counterintuitive, by borrowing from electrostatics concepts. Here the electric field would be represented by the gradient of a potential. As the spheres become more conducting (i.e., larger $n k$ ), the internal potential within each sphere would become increasingly more uniform. When the spheres are aligned parallel to the incident electric field (i.e., in the direction of the incident potential gradient), the highly conducting spheres would attain uniform yet different values of internal potential. At the contact point between the spheres one would thus find a significant gradient in external potential. Essentially, the flux (represented by the electric field) gets concentrated at the contact point. Consequently, the field inside each sphere may be uniform, yet the external field adjacent to the spheres can be highly nonuniform. As a result, several expansion orders are required for resolution of the field about the contact point.

It should also be noted that the required number of orders given for $x=0.1$ does not decrease as the size parameters become smaller. However, the magnetic (TE) modes in the spheres become negligible in comparison with the electric (TM) modes for small size parameters, and we could reduce the equations in this case by neglecting the TE contributions in the expansions. Nevertheless, severe numerical problems arise when one attempts to calculate the cross sections of small, highly conducting sphere clusters, in that it is necessary to calculate Bessel functions of appreciable order for small arguments. An alternative approach would be to apply an electrostatics analysis to the sphere cluster, which would be valid when the overall size of the cluster is significantly smaller than the radiation wavelength. ${ }^{24}$

The calculation results presented here have significant implications in the modeling of extinction and absorption of sphere clusters such as soot aggregates. Because the diameters of the primary spheres constituting the aggregate are typically much smaller than visible and IR wavelengths, several previous modeling efforts have started with a dipole approximation of the spheres. ${ }^{10,12,13} \mathrm{Re}$ sults indicate that the dipole approximation can seriously underestimate the cross sections of clusters for large $n k$. This is illustrated in Fig. 2, where the absorption cross section of a two-sphere cluster, divided by that for independent spheres, is plotted versus the number of expansion orders for three values of refractive index. The size parameter of the spheres in the cluster is 0.01 , and the results are for random orientation of the cluster. For $m=1.6+0.6 i$, which is appropriate for carbonaceous soot in the visible, a dipole model $\left(N_{t}=1\right)$ provides a good estimate of the absorption, yet the increase in absorption for the cluster over that for independent spheres is not especially significant. Once the refractive index increases to $2+1 i$ and $3+2 i$, which would be appropriate for soot in the near to far IR, both the increase in absorption of the cluster relative to independent spheres and the error in using a dipole model increase markedly.

Soot aggregates formed in combustion processes are rarely simple pairs of spheres. Depending on the residence time in the flame and the particle concentration, the aggregates can contain from tens to thousands of primary spheres. ${ }^{1}$ It is obviously of interest to determine whether the conclusions obtained for a binary cluster carry over to a cluster of several spheres. To examine this, we performed calculations on fractallike clusters of spheres. When they are formed from diffusion-limited aggregation (DLA), soot aggregates have a structure that

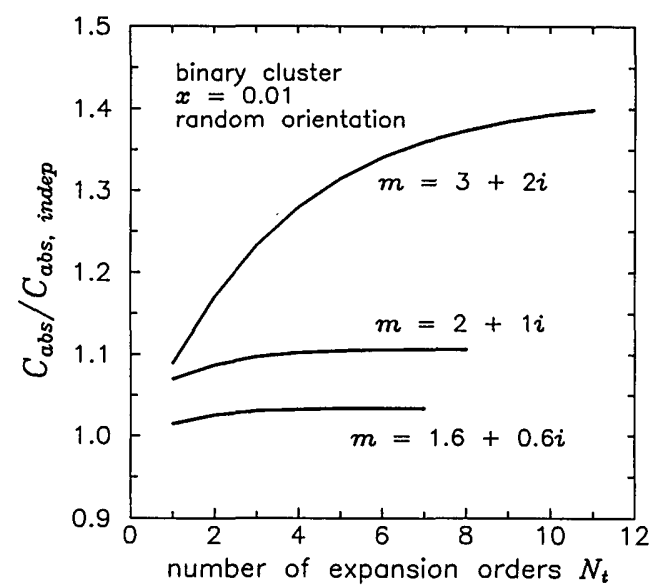

Fig. 2. Random-orientation absorption cross section of a two-sphere cluster, divided by that for independent spheres, versus the number of orders in the scattered-field expansion. 


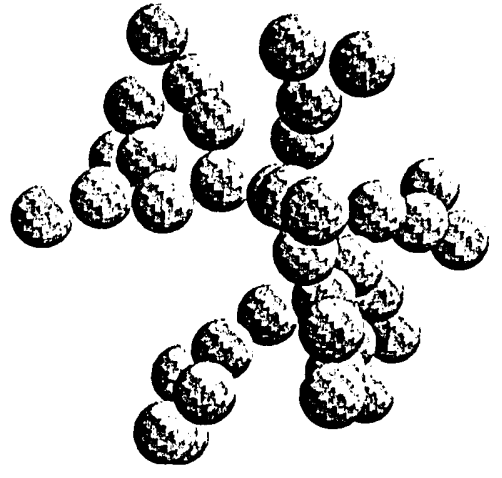

Fig. 3. 40-sphere fractal cluster generated with the sequential algorithm.

can be represented by ${ }^{25,26}$

$$
N_{s}=k_{f}\left(\frac{R_{g}}{d_{p}}\right)^{D_{f}}
$$

where $d_{p}$ is the primary sphere diameter, $R_{g}$ is the radius of gyration, $D_{f}$ is the fractal dimension, and $k_{f}$ is a prefactor constant. For a specified number of equal-sized spheres in the cluster, $R_{g}$ is given by

$$
R_{g}{ }^{2}=\frac{1}{N_{s}} \sum_{i=1}^{N_{s}} r_{i}{ }^{2},
$$

where $r_{i}$ is the distance from sphere $i$ to the center of mass of the cluster. Rather than performing numerical DLA simulations to obtain the particle positions in a cluster, we developed a sequential algorithm that mimicks Eq. (49). Beginning with a pair of contacting spheres and specified values of $k_{f}$ and $D_{f}$, a third sphere was randomly attached to the surface of one of the two spheres, with the constraint that the radius of gyration calculated for the three-sphere cluster exactly satisfy Eq. (49). The process was then repeated for the fourth, fifth, and additional spheres. Values of $k_{f}=5.6$ and $D_{f}=1.9$ were used in the calculations, which correspond to values obtained from direct DLA simulations. ${ }^{26}$ The properties of the aggregates generated with this algorithm obeyed the known statistical relationships of DLA aggregates, ${ }^{25}$ yet the positions could be calculated in a fraction of the time required for DLA simulations. An illustration of a 40sphere cluster generated from the sequential algorithm is given in Fig. 3.

Using the multipole formulation, we calculated radiative extinction and absorption properties of the 40-sphere fractal cluster in Fig. 3, and results appear in Figs. 4 and 5. Presented are the random-orientation cluster extinction efficiency $\bar{Q}_{\text {ext }}$ and absorption efficiency $\bar{Q}_{\mathrm{abs}}$, divided by the cluster volume mean size parameter $x_{V}=x_{p} N_{s}{ }^{1 / 3}$, versus the primary sphere size parameter $x_{p}$. In Fig. 4 the sphere refractive index is $m=1.6+0.6 i$, and in Fig. 5 it is $m=2+1 i$. The number of expansion orders used in the calculations was $N_{t}=2$ and 3 , respectively. The efficiency $\bar{Q}$ is defined according to the volume mean radius $a_{V}$, so that $\bar{C}=\pi a_{V}^{2} \bar{Q}$. The quantity $Q / x_{V}$ is thus proportional to $C / V$, and in the limit $x_{V} \rightarrow 0$ this ratio will become constant. Also given are results for the same refractive indices that we obtained by including only the first electric dipole term in the calculations-which corresponds to a discrete dipole approximation of the cluster-along with the extinction and the absorption predicted from Lorenz/Mie theory for an equivalent sphere having the same refractive index as that of the spheres and a size parameter of $x_{V}$. This latter model has been suggested by Drolen and $\operatorname{Tien}^{27}$ as a good approximation for the cross sections of soot clusters.

The computational overhead in calculating the exact orientation-averaged cross sections is considerable. Since the number of scattering coefficients for the cluster will be $2 N_{s} N_{t}\left(N_{t}+2\right)$, calculation of cluster extinction and absorption for the second refractive index would involve inversion of a $1200 \times 1200$ complex-valued matrix along with subsequent matrix multiplication. The exact orientation-averaging formulation is perhaps not the most efficient means to obtain the averaged cross sections for fractal clusters of small spheres. Because of the random structure of the clusters the cross sections are not strongly dependent on the direction and the polarization of the incident radiation. In addition, the equations for the scattering coefficients for a fixed orientation converge rapidly with conjugate gradient iteration methods. It

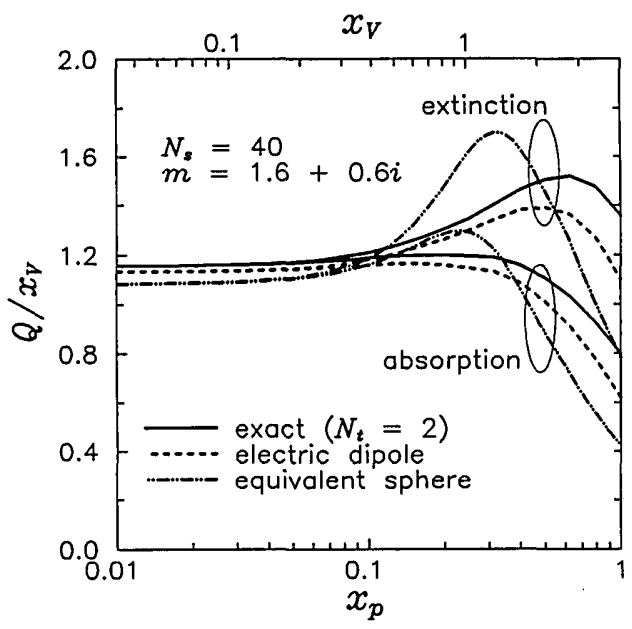

Fig. 4. Ratio of cluster efficiency and volume equivalent size parameter $x_{V}$ versus size parameter for exact $\left(N_{t}=2\right)$, electric dipole, and volume equivalent-sphere predictions. The refractive index is $m=1.6+0.6 i$.

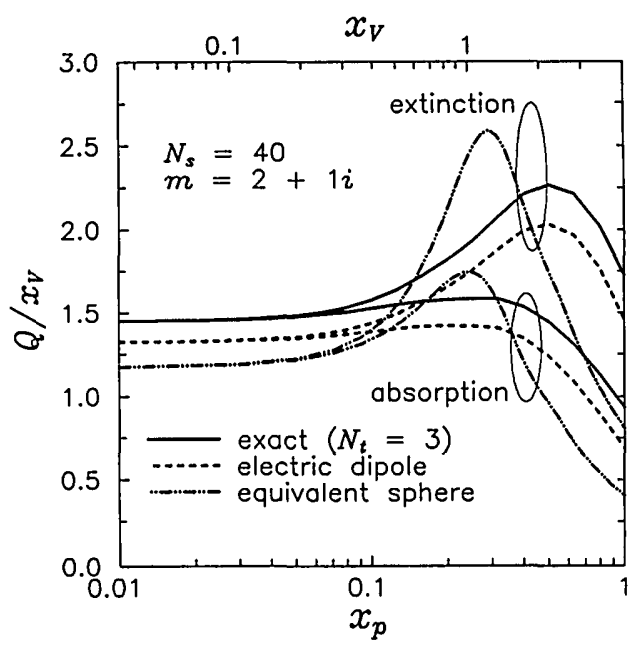

Fig. 5. Same as Fig. 4 but with $m=2+1 i$. 
thus makes more sense to calculate the averaged cross sections by a suitable quadrature of the fixed-orientation cross sections over incident directions. This approach was taken in calculation of the cross sections of the 40sphere cluster. We used Gaussian quadrature to integrate over $\beta$, and the trapezoid rule was used for $\alpha$ and $\gamma$. Four points each for $\alpha$ and $\beta$ and two points for $\gamma$ were required for $0.1 \%$ accuracy in the calculated cross sections.

Results for $m=1.6+0.6 i$ indicate that all three formulations (multipole, dipole, and equivalent sphere) agree well for small volume mean size parameter $x_{V}$. Once $x_{V}$ increases to near unity, the equivalent-sphere and multiple-sphere models predict significantly different trends. The equivalent sphere shows a strong resonant extinction and absorption for $x_{V} \approx 1$, whereas the resonance is not nearly so strong in the dipole and multipole results. In addition, the location of the maximum extinction for the dipole and multipole results occurs for $x_{V}$ approximately equal to 2.5, and for larger $x_{V}$ the equivalent-sphere and multipole predictions are significantly different. When $m=2+1 i$, the discrepancy in the three formulations becomes more apparent over the entire range of size parameters. The equivalent-sphere model significantly underpredicts absorption for small $x_{V}$ and overpredicts the resonant extinction and absorption occurring at $x_{V} \approx 1$. Note that in both Figs. 3 and 4 the ratio of the dipole and multipole absorption results is roughly constant over a wide range of size parameters. This suggests that results from the dipole model, which are considerably easier to compute than the multipole results, could be corrected by a constant empirical factor that would depend on the sphere refractive index and the structure of the cluster.

Whether the analytical equations for orientationaveraged cross sections are of practical utility is open to question. The main stumbling block to their application is the need to obtain the $T$ matrix, which can involve numerical inversion of rather large matrices. Perhaps a more useful application of the orientation-averaged formulation is in the examination of simple pairs of spheres. As was mentioned above, the azimuthal decoupling that results when spheres share a common $z$ axis results in a considerable reduction in the order of the equations and makes feasible the numerical inversion process. We can obtain a further reduction in order for sphere pairs by formally factoring out one of the sets of scattering coefficients in Eq. (13). If we eliminate, for example, the coefficients $\mathbf{a}_{m n p}{ }^{1}$ in Eq. (13), the following system is obtained for $\mathbf{a}_{m n p}{ }^{2}$ :

$$
\begin{aligned}
& \left(I-\overline{\mathbf{a}}_{n p}{ }^{2} H_{m n p m n^{\prime} p^{\prime}}{ }^{21} \overline{\mathbf{a}}_{n^{\prime} p^{\prime}}{ }^{1} H_{m n^{\prime} p^{\prime} m l q}{ }^{12}\right) \mathbf{a}_{m l q}{ }^{2} \\
& =\overline{\mathbf{a}}_{n p}{ }^{2}\left(\mathbf{p}_{m n}{ }^{2}-H_{m n p m n^{\prime} p^{\prime}}{ }^{21} \overline{\mathbf{a}}_{n^{\prime} p^{\prime}}{ }^{1} \mathbf{p}_{m n^{\prime}}{ }^{1}\right)
\end{aligned}
$$

Note that the decoupling of degree $m$ is implicit in the above equation, and the sum is taken over orders $l$ and $n^{\prime}$ and modes $q$ and $p^{\prime}$. The $T$-matrix elements for sphere 2 are readily identified as

$$
\begin{aligned}
& T^{22}=\left(I-\overline{\mathbf{a}}^{2} H^{21} \overline{\mathbf{a}}^{1} H^{12}\right)^{-1} \overline{\mathbf{a}}^{2}, \\
& T^{21}=-T^{22} H^{21} \overline{\mathbf{a}}^{1}
\end{aligned}
$$

and the elements for sphere 1 are given in terms of $T^{22}$ by

$$
\begin{aligned}
& T^{12}=-\overline{\mathbf{a}}^{1} H^{12} T^{22}, \\
& T^{11}=\overline{\mathbf{a}}^{1}\left(I+H^{12} T^{22} H^{21} \overline{\mathbf{a}}^{1}\right) .
\end{aligned}
$$

It would obviously make the most sense to choose sphere 2 so it were the smaller of the two and thus would require fewer orders in the field expansion. The order of the inverted matrix in Eq. (52) will thus be minimized.

With the use of Eq. (32) the orientation-averaged extinction cross section of sphere 2 is given by

$$
\begin{aligned}
\bar{C}_{\mathrm{ext}}^{2}= & \frac{2 \pi}{\hbar^{2}} \operatorname{Re}\left(T_{m n p m n p}{ }^{22}-T_{m n p m n^{\prime} p^{\prime}}{ }^{22}\right. \\
& \left.\times H_{m n^{\prime} p^{\prime} m l q}{ }^{21} \overline{\mathbf{a}}_{l q}{ }^{1} J_{m l q m n p}{ }^{12}\right)
\end{aligned}
$$

One can develop a relatively simple formula for the orientation-averaged absorption cross section of sphere 2 by combining Eqs. (46), (52), and (53) to yield

$$
\bar{C}_{\mathrm{abs}}{ }^{2}=\frac{2 \pi}{\hbar^{2}} \operatorname{Re}\left(S_{m n p m n p}{ }^{2}+S_{m n p m l q}{ }^{2} U_{m n p m l q}{ }^{2}\right),
$$

where the matrices $S$ and $U$ are defined by

$$
\begin{aligned}
S_{m n p m l q}{ }^{2}= & E_{m n^{\prime}} \overline{\mathbf{d}}_{n^{\prime} p^{\prime}}{ }^{2} T_{m n^{\prime} p^{\prime} m n p}{ }^{22} T_{m n^{\prime} p^{\prime} m l q}{ }^{2 * *} / E_{m l}, \\
U_{m n p m l q}{ }^{2}= & E_{m l} \overline{\mathbf{a}}_{n^{\prime} p^{\prime}}{ }^{1} H_{m n p m n^{\prime} p^{\prime}}{ }^{21}\left(H_{m l q m n^{\prime} p^{\prime}}{ }^{21 *} \overline{\mathbf{a}}_{n^{\prime} p^{\prime}}{ }^{1 *}\right. \\
& \left.-2 J_{m l q m n^{\prime} p^{\prime}}{ }^{2 *}\right) / E_{m n^{\prime}} .
\end{aligned}
$$

Equations for the orientation-averaged extinction and absorption of sphere 1 are obtained from Eqs. (56) and (57) by interchange of the superscripts 1 and 2 . Of course, if the spheres were equal, so would be their averaged cross sections. For the specific case of a pair of identical electric dipoles separated by a distance $R^{12}$ the $T$ matrix can be calculated analytically, which leads to the relatively simple formulas

$$
\begin{aligned}
& \bar{C}_{\text {ext }, 2, d p}=\frac{4 \pi}{\hbar^{2}} \\
& \times \operatorname{Re}\left(\bar{a}_{1}\left\{3-\bar{a}_{1}\left[\frac{2 g_{1}\left(f_{1}-\bar{a}_{1} g_{1}\right)}{1-\left(\bar{a}_{1} g_{1}\right)^{2}}+\frac{g_{2}\left(f_{2}-\bar{a}_{1} g_{2}\right)}{1-\left(\bar{a}_{1} g_{2}\right)^{2}}\right]\right\}\right), \\
& \bar{C}_{\mathrm{abs}, 2, d p}=\frac{4 \pi}{\hbar^{2}} \bar{d}_{1}\left|\bar{a}_{1}\right|^{2} \\
& \times \operatorname{Re}\left[\left(3-\bar{a}_{1}\left\{\frac{2 g_{1}\left[\left(\bar{a}_{1} g_{1}\right)^{*}\left(\left|\bar{a}_{1} g_{1}\right|^{2}-1\right)+2\left(f_{1}-\bar{a}_{1} g_{1}\right)\right]}{\left|1-\left(\bar{a}_{1} g_{1}\right)^{2}\right|^{2}}\right.\right.\right. \\
& \left.\left.\left.+\frac{g_{2}\left[\bar{a}_{1} g_{2} *\left(\left|\bar{a}_{1} g_{2}\right|^{2}-1\right)+2\left(f_{2}-\bar{a}_{1} g_{2}\right)\right]}{\left|1-\left(\bar{a}_{1} g_{2}\right)^{2}\right|^{2}}\right\}\right)\right],
\end{aligned}
$$

where $\bar{a}_{1}$ and $\bar{d}_{1}$ are defined in Eqs. (11) and (41) and the quantities $f$ and $g$ depend only on the separation distance between the spheres and are given by

$$
\begin{aligned}
& g_{1}=h_{0}\left(k R^{12}\right)-1 / 2 h_{2}\left(k R^{12}\right), \\
& g_{2}=h_{0}\left(k R^{12}\right)+h_{2}\left(\hbar R^{12}\right), \\
& f_{1}=j_{0}\left(k R^{12}\right)-1 / 2 j_{2}\left(k R^{12}\right), \\
& f_{2}=j_{0}\left(k R^{12}\right)+j_{2}\left(\hbar R^{12}\right) .
\end{aligned}
$$

Note that the first term in Eqs. (60) and (61) represents the extinction and the absorption for the isolated spheres, 
and the subsequent terms provide the corrections for field interactions between the spheres.

In closing, it should be mentioned that the results presented here have not attempted to address the angular dependence of the scattered radiation from the sphere cluster (i.e., the differential scattering cross sections). Unlike the case for the total cross sections, it does not appear possible to express the differential cross sections for random orientation in terms of relatively simple expressions involving the matrix element $T^{i j}$. That this is the case is not surprising. As was mentioned above, the number of orders in the vector harmonic expansion representing the total scattered field from the cluster will be greater than the number of orders representing the fields scattered from the individual spheres. Although these higher orders (resulting from dependent scattering effects) were seen to disappear from the expressions for the total cross sections, they must be accounted for in the differential scattering cross sections. The transformation of the $T$ matrix into the $T^{0}$ matrix is thus unavoidable in formulating the orientation-averaged differential cross sections. The reader is referred to the recent papers by Khlebtsov ${ }^{20}$ and Mishchenko ${ }^{21}$ for the analysis of orientation-averaged scattering quantities from the $T^{0}$-matrix representation.

\section{APPENDIX A: ADDITION COEFFICIENTS}

Although explicit relations exist for the addition coefficients $A_{k l m n}$ and $B_{k l m n},{ }^{28}$ these coefficients are most efficiently calculated from recurrence relations. Details of the derivation of the recurrence relations can be found in Mackowski ${ }^{8}$; only the working relations are given here. The vector harmonic addition coefficients can be calculated from the scalar harmonic addition coefficients $C_{k l m n}$ through

$$
\begin{aligned}
A_{k l m n}^{(\nu)}= & \frac{1}{2 n(n+1)}\left[(n-m)(n+m+1) C_{k+1 l, m+1 n}^{(\nu)}\right. \\
& \left.+2 m k C_{k l m n}^{(\nu)}+(l+k)(l-k+1) C_{k-1 l, m-1 n}^{(\nu)}\right] \\
B_{k l m n}^{(\nu)}= & -i \frac{2 n+1}{2 n(n+1)(2 n-1)}[(n-m)(n-m-1) \\
& \times C_{k+1 l ; m+1, n-1}^{(\nu)}+2 k(n-m) C_{k l, m n-1}^{(\nu)}-(l+k) \\
& \left.\times(l-k+1) C_{k-1 l ; m-1, n-1}^{(\nu)}\right]
\end{aligned}
$$

The scalar addition coefficients, in turn, can be obtained from the recurrence relations

$$
\begin{aligned}
\frac{1}{2 l+1}[ & \left.C_{k l-1, m n}^{(\nu)}+C_{k l+1, m n}^{(\nu)}\right] \\
= & \frac{1}{2 n-1} C_{k-1 l ; m-1, n-1}^{(\nu)}+\frac{1}{2 n+3} C_{k-1 l ; m-1, n+1}^{(\nu)}, \\
\frac{1}{2 l+1}\left[(l+k)(l+k+1) C_{k l-1, m n}^{(\nu)}\right. & \left.+(l-k)(l-k+1) C_{k l+1, m n}^{(\nu)}\right] \\
= & \frac{(n-m)(n-m-1)}{2 n-1} C_{k+1 l ; m+1, n-1}^{(\nu)} \\
& +\frac{(n+m+1)(n+m+2)}{2 n+3} C_{k+1 l ; m+1, n-1}^{(\nu)},
\end{aligned}
$$

$$
\begin{aligned}
\frac{1}{2 l+1}[(l & \left.+k) C_{k l-1, m n}^{(\nu)}-(l-k+1) C_{k l+1, m n}^{(\nu)}\right] \\
& =-\frac{n-m}{2 n-1} C_{k l, m n-1}^{(\nu)}+\frac{n+m+1}{2 n+3} C_{k l, m n+1}^{(\nu)} .
\end{aligned}
$$

In the above equations it is understood that the addition coefficients $A_{k l m n}, B_{k l m n}$, and $C_{k l m n}$ are zero if $|k|>l$ or $|m|>n$.

Starting values for the above recurrence relations are obtained explicitly from the formula

$$
\begin{aligned}
& C_{00 m n}^{(\nu)} \\
& \quad=\left\{\begin{array}{r}
(-1)^{n+m}(2 n+1) j_{n}\left(k R^{i j}\right) P_{n}^{-m}\left(\cos \Theta^{i j}\right) \exp \left(-i m \Phi^{i j}\right) \\
\nu=1 \\
(-1)^{n+m}(2 n+1) h_{n}\left(k R^{i j}\right) P_{n}^{-m}\left(\cos \Theta^{i j}\right) \exp \left(-i m \Phi^{i j}\right) \\
\nu=3
\end{array}\right.
\end{aligned}
$$

where the angles $\Theta^{i j}$ and $\Phi^{i j}$ denote the direction of origin $i$ relative to origin $j$. It should be noted that the recurrence relations in Eqs. (A3)-(A5) are unconditionally stable. However, to calculate $C_{k l m n}$ up to $l=n=N_{t}$ orders, one must begin with $C_{00 m n}$ calculated to $n=2 N_{t}$ orders.

The addition coefficients $A_{k 1 m n}, k=-1,0,1$, are involved in the computations of sphere clusters in which the size parameters of the spheres are significantly less than unity (i.e., the Rayleigh approximation). Explicit relations for these coefficients are

$$
\begin{aligned}
A_{-11 m n}= & \frac{(-1)^{m+n}}{2 n(n+1)}\left[(n+1)(n-m)(n-m-1) u_{-m-1, n-1}\right. \\
& \left.-n(n+m+1)(n+m+2) u_{-m-1, n+1}\right], \\
A_{01 m n}= & \frac{-(-1)^{m+n}}{n(n+1)}\left[(n+1)(n-m) u_{-m, n-1}\right. \\
& \left.-n(n+m+1) u_{-m, n+1}\right], \\
A_{11 m n}= & \frac{(-1)^{m+n}}{n(n+1)}\left[(n+1) u_{-m+1, n-1}-n u_{-m+1, n+1}\right],
\end{aligned}
$$

where $u_{m n}$ denotes the scalar spherical harmonic,

$$
u_{m n}=z_{n}\left(R^{i j}\right) P_{n}{ }^{m}\left(\cos \Theta^{i j}\right) \exp \left(i m \Phi^{i j}\right),
$$

and $z_{n}$ refers to $j_{n}$ or $h_{n}$.

\section{ACKNOWLEDGMENTS}

The author wishes to acknowledge helpful discussions and collaborations with P. Flatau and K. Fuller.

\section{REFERENCES}

1. R. A. Dobbins and C. M. Megaridis, "Morphology of flamegenerated soot as determined by thermophoretic sampling," Langmuir 3, 254-259 (1987).

2. J. H. Brunning and Y. T. Lo, "Multiple scattering of EM waves by spheres. Part I. Multipole expansion and rayoptical solutions," IEEE Trans. Antennas Propag. AP-19, 378-390 (1971).

3. F. Borghese, P. Denti, R. Saija, G. Toscano, and O. I. Sindoni, "Multiple electromagnetic scattering from a cluster of spheres. I. Theory," Aerosol Sci. Technol. 3, 227-235 (1984). 
4. K. A. Fuller and G. W. Kattawar, "Consummate solution to the problem of classical electromagnetic scattering by an ensemble of spheres: I. Linear chains," Opt. Lett. 13, 90-92 (1988)

5. K. A. Fuller and G. W. Kattawar, "Consummate solution to the problem of classical electromagnetic scattering by an ensemble of spheres: II. Clusters of arbitrary configuration," Opt. Lett. 13, 1063-1065 (1988).

6. K. A. Fuller, "Optical resonances and two-sphere systems," Appl. Opt. 33, 4716-4731 (1991).

7. K. A. Fuller, "Scattering and absorption cross sections of compounded spheres. I. Theory for external aggregation," J. Opt. Soc. Am. A (to be published).

8. D. W. Mackowski, "Analysis of radiative scattering for multiple sphere configurations," Proc. R. Soc. London Ser. A 433, 599-614 (1991)

9. D. S. Saxon, "Lectures on the scattering of light," Department of Meteorology Sci. Rep. 9 (University of California at Los Angeles, Los Angeles, Calif., 1955).

10. A. R. Jones, "Electromagnetic wave scattering by assemblies of particles in the Rayleigh approximation," Proc. R. Soc. London Ser. A 366, 111-127 (1979).

11. B. T. Draine, "The discrete-dipole approximation and its application to interstellar graphite grains," Astrophys. J. 333, 848-872 (1988)

12. M. F. Iskander, H. Y. Chen, and J. E. Penner, "Optical scattering and absorption by branched chains of aerosols," Appl. Opt. 28, 3083-3091 (1989).

13. J. C. Ku and K.-H. Shim, "A comparison of solutions for light scattering and absorption by agglomerated or arbitrarilyshaped particles," J. Quant. Spectrosc. Radiat. Transfer 47, 201-220 (1992).

14. F. Borghese, P. Denti, R. Saija, G. Toscano, and O. I. Sindoni, "Macroscopic optical constants of a cloud of randomly oriented nonspherical scatterers," Nuovo Cimento B 81, 29-50 (1984).

15. C. F. Bohren and D. R. Huffman, Absorption and Scattering of Light by Small Particles (Wiley, New York, 1983).
16. P. C. Waterman, "Symmetry, unitarity, and geometry in electromagnetic scattering," Phys. Rev. D 3, 825-839 (1971).

17. P. W. Barber and C. Yeh, "Scattering of electromagnetic waves by arbitrary shaped dielectric bodies," Appl. Opt. 14, 2864-2872 (1975).

18. F. Borghese, P. Denti, R. Saija, and O. I. Sindoni, "Reliability of the theoretical description of electromagnetic scattering from nonspherical particles," J. Aerosol Sci. 20, 1079-1081 (1989).

19. A. R. Edmunds, Angular Momentum in Quantum Mechanics (Princeton U. Press, Princeton, N.J., 1957).

20. N. G. Khlebtsov, "Orientational averaging of light-scattering observables in the T-matrix approach," Appl. Opt. 31, 5359-5365 (1992).

21. M. I. Mishchenko, "Light scattering by randomly oriented axially symmetric particles," J. Opt. Soc. Am. A 8, 871-882 (1991).

22. R. T. Wang, J. M. Greenberg, and D. W. Schuerman, "Experimental results of dependent light scattering by two spheres," Opt. Lett. 6, 543-545 (1981).

23. W. J. Wiscombe, "Improved Mie scattering algorithms," Appl. Opt. 19, 1505-1509 (1980)

24. J. M. Gerardy and M. Ausloos, "Absorption spectrum of clusters of spheres from the general solution of Maxwell's equations: the long wavelength limit," Phys. Rev. B 22, 4950-4959 (1980).

25. R. Jullien and R. Botet, Aggregation and Fractal Aggregates (World Scientific, Singapore, 1987).

26. R. D. Mountain and G. W. Mulholland, "Light scattering from simulated smoke agglomerates," Langmuir 4, 1321-1326 (1988).

27. B. L. Drolen and C. L. Tien, "Absorption and scattering of agglomerated soot particles," J. Quant. Spectrosc. Radiat. Transfer 37, 433-448 (1987).

28. O. R. Cruzan, "Translational addition theorems for spherical vector wave functions," Q. Appl. Math. 20, 33-39 (1962). 